\title{
Mechanism Design and Motion Analysis of a Flapping-Wing Air Vehicle
}

\author{
Yunde Shi $\mathbb{D}^{1},{ }^{1}$ Wang He, ${ }^{1}$ Mingqiu Guo, ${ }^{1}$ Dan Xia $\mathbb{D}^{\circ},{ }^{1}$ Xiang Luo, ${ }^{1}$ and Xiaoqiang Ji ${ }^{2}$ \\ ${ }^{1}$ School of Mechanical Engineering, Southeast University, Nanjing 211189, China \\ ${ }^{2}$ Shenzhen Institute of Artificial Intelligence and Robotics for Society, The Chinese University of Hongkong, \\ Shenzhen 518172, China \\ Correspondence should be addressed to Dan Xia; dxia@seu.edu.cn
}

Received 29 November 2021; Accepted 26 January 2022; Published 4 March 2022

Academic Editor: Adnan Maqsood

Copyright (C) 2022 Yunde Shi et al. This is an open access article distributed under the Creative Commons Attribution License, which permits unrestricted use, distribution, and reproduction in any medium, provided the original work is properly cited.

Micro flapping-wing air vehicles (FWAV) are highly appreciated by scientists and scholars around the world due to their small size, high flexibility, and better manoeuvrability. There are huge potential applications of flapping-wing robots in rescue, survey, and search, as they can enter more dangerous and narrow spaces to accomplish tasks that were previously impossible for people. In this paper, we first analyze the flapping motion of birds by reviewing a large amount of information and literature and identifying the sources of lift and thrust during flight. Then, the double crank and double rocker mechanisms are optimized as the flapping mechanism. Proper flapping-wing size is further determined by analyzing the relative position between the driving mechanism and the flapping mechanism. Moreover, a pitch mechanism consisting of ball hinges is designed to realize the wing's pitching motion for additional thrust. The fuselage and landing gear of the flapping-wing robot are designed for low air resistance and lightweight, and the tail mechanism are designed to have both roll and pitch freedoms, offering excellent manoeuvrability during flight. Simulation results show positive lift force is generated based on the novel pitching design for the small wing. The research in this paper is an important resource for novice researchers to design and study the advanced and complex problems of flappingwing air vehicles.

\section{Introduction}

A micro air vehicle (MAV) is a mechanical device with the ability to fly and can be divided into three types according to the way it generates lift: a fixed-wing flying robot like a jet-propelled aircraft, a rotor-wing vehicle like a helicopter, and a flappingwing robot that mimics birds or insects $[1,2]$. Micro flappingwing air vehicles have many advantages over the first two types of flight, including their ultra-small size, high flexibility, better manoeuvrability, and excellent hovering ability [3]. These advantages provide MFWAV with the ability of obstacle avoidance and navigation control in tight spaces. The use of MFWAV can perform many tasks that were previously difficult for people to accomplish $[4,5]$, for example, search and reconnaissance, communication, transportation.

The microbat, a representative bat-like micro flying robot developed by Caltech, is battery-powered and has a titanium alloy skeleton with a polymer film covering the wings [6-8]. The rotation of the micromotor is transformed into an up-anddown flapping motion of both wings through a transmission mechanism, which generates sufficient lift and thrust. The direction and flight attitude are controlled by a tail composed of a flat tail and a drooping tail. The vehicle has been successfully tested flown $[9,10]$.

Scientists at Festo in Germany have developed a miniature wing-flapping robot called "Smart Bird", which can imitate the flight of birds and flap its wings in an extremely realistic manner [11]. There are two rotating wheels to control the wings' up-anddown motion. The angle of the wings can also be adjusted by the installed torque motor, so it has very good aerodynamic performance and high sensitivity. The Smart Bird's flight is controlled via a radio control unit, and it is also possible to operate on automatic mode so that the Smart Bird can soar through the air on its own [12]. 
Michelson, an engineer at the Georgia Institute of Technology, developed the "Entomopter", a miniature insect-like wing-flapping robot based on the flight principles of insects [13, 14]. This flying machine is designed with a special structure and materials to imitate the butterfly's flapping motion, powered by the chemical muscle drive. The drive can be highly efficient in reciprocating motion for the wings to flap up and down to generate sufficient lift [15]. At present, many scholars believe that when the size of micro flying robots is further reduced to near insect size, the use of conventional components such as rotary motors, bearings, and connecting rods will make the whole machine inefficient [16]. Therefore, a group of biomimetic wing-flapping robots with efficient reciprocating drives are born, represented by Entomopter. It adopts a front-to-back cross-flapping layout, giving it hovering capability, balance, and controllability.

The research team led by Prof. Haisong Ang of Nanjing University of Aeronautics and Astronautics has been conducting research on flutter flight since 2004 [17]. In the process of observing and recording the flight of birds, many mysteries of fluttering flight were discovered, and a bionic fluttering aircraft bird with the autonomous flight was successfully developed in 2011. The miniature wing-flapping robotic bird has a wingspan of about $60 \mathrm{~cm}$, weighs about $50 \mathrm{~g}$, and resembles a common large bird [18-20]. The bird is powered by a 15-gram battery and a miniature motor, and the motor's power is transferred to the wings, which mimic the flap of a bird's wings, and the flutter has been successfully tested [21, 22].

Aiming at structural simplicity, motion symmetry, and flapping efficiency, this paper proposes an optimized double crank and double rocker mechanism as the flapping mechanism. Proper flapping-wing size is determined by analyzing the relative position between the driving mechanism and the flapping mechanism. Moreover, a novel pitch mechanism consisting of ball hinges is designed to realize the small wing's pitching motion for additional thrust. The fuselage and landing gear of the flapping-wing robot are designed for low air resistance and lightweight, and the tail mechanism is designed to have both roll and pitch freedoms, offering advanced manoeuvrability during flight. Simulation results show faster flapping motion with larger wingspan area during the downstroke than upstroke, for positive aerodynamic lift. Additionally, the novel pitching design for the small wing further improves the lift forces during flight. The research in this paper is an important resource for novice researchers to design and study the advanced and complex problems of flapping-wing air vehicles.

The rest of the paper is presented below. In Section 2, bionic flight mechanisms and design requirements are described in detail. In Section 3, the flap wing structure design is discussed and analyzed. In Section 4 , the overall design of the flapping-wing robot is illustrated, and simulation is run for the flapping motion, which shows positive lift force during flight. Section 5 gives the main conclusions of the paper.

\section{Bionic Flight Mechanisms and Design Requirements}

This paper focuses on the design of a micro flapping-wing robot based on the study of the flapping mechanism of birds and the application of bionic design principles.

2.1. Bionic Flight Mechanisms. Birds usually have streamlined bodies to reduce the drag caused during flight. The source of both lift and thrust is from the up-and-down flapping motion of the wings. During the flight, the folding of the wings can change the wingspan area and thus control the interaction forces with the air. Also, the wings can make a pitching motion about the leading edge of the wings, thus changing the direction of the force and creating the thrust component. During the flight of birds, the typical state of flapping action is illustrated as shown in Figure 1.

By analysis, the 14 decomposed movements during the above flutter can be classified into four parts (strokes).

(1) Down stroke (Figure 1, 1-5): The large wing and small wing move together from the highest point of the small wing downward until they reach the lowest point of the large wing. During this stroke, the large and small wings are in the same line, and the wingspan is at its maximum.

(2) Folding stroke (Figure 1, 6-10): The large wing remains basically motionless, and the small wing continues to move downward from the end state of the previous stroke until it reaches the lowest point. In this process, the large and the small wing go through a process from collinear to folding.

(3) Lift stroke (Figure 1, 11-13): In this process, the angle between the large wing and the small wing is basically kept constant, and the whole wing moves upward until it reaches the highest point of the large wing. The large wing and small wing remain folded.

(4) Unfolding stroke (Figure 1, 14): In this process, the large wing remains basically unchanged, and the small wing continues to move upward until it reaches the highest point. During this stroke, the wing changes from folded to collinear.

The above analysis, however, only demonstrates the principle of birds' flapping in an intuitive manner. In fact, the four strokes are not completely independent. From the above analysis, it can be seen that during the flight of the bird, the wings do not simply flap up and down, but are accompanied by the associated angular change between the two subwings.

Along with the up-and-down flapping motion, the wings also make a pitching motion around the wing's leading edge. During the downward stroke, the wings rotate upward, causing the back part of the wings to be slightly higher than the front part of the wings against the body. This interaction with the air is perpendicular to the lower surface of the wing, that is, diagonally upward, creating a thrust component. Similarly, during the upward lift, the wings rotate slightly 

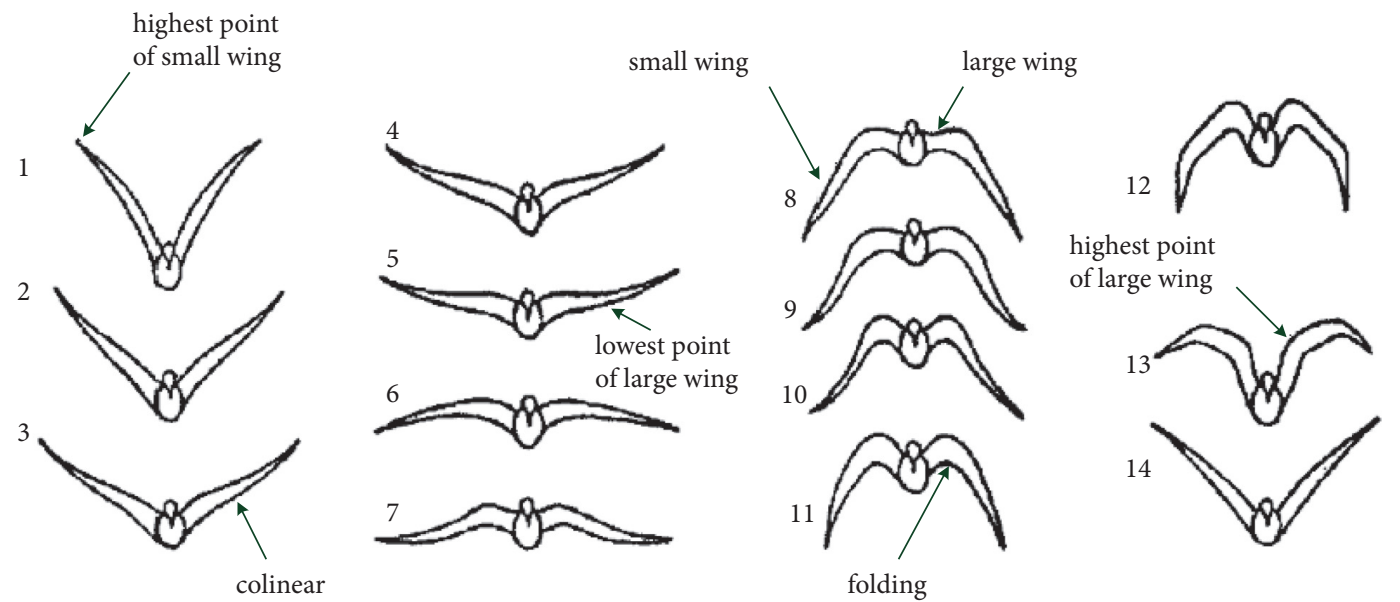

Figure 1: Bird flapping cycle.

downward, generating both forward and downward forces [23]. When we designed the micro flapping prototype, specific mechanisms are needed to achieve this bionic flapping movement.

In addition to flapping, the role of the bird's tail in the flight process is crucial. The tail of a micro flapping-wing air vehicle is equivalent to the tail of a modern aircraft. The MFWAV's agility is created by the synergy of the wings and the tail [24]. These two work together to allow MFWAVs to change their flight attitude in the air whenever needed, for a variety of movements, such as, turns, dives, flips.

2.2. Design Requirements. After the above analysis of the bionic flight mechanism of birds, the requirements for the design of micro flapping-wing robotics are as follows:

(1) The size of all parts of the MFWAV is reasonable, and the structure is hollow.

(2) The shape of the fuselage follows the streamlined design, minimizing the drag during flight.

(3) There is only one pair of symmetrical wings, which can be folded. Rhythmic fluttering motion should achieve in flight. The wings unfold as much as possible during downward flight and fold as much as possible during upward stroke, for maximized lift and thrust.

(4) The small wing can pitch around the leading edge of the large wing and provide thrust during the corresponding flapping process.

(5) The tail wing has two degrees of freedom, including pitch and roll, for attitude control of the MFWAV.

\section{Structure Design of Flap Wing}

3.1. Selection of Flapping-wing Mechanism. In order to transform the rotating action of the rotary motor into the function of upward and downward fluttering, the design in this paper uses a linkage mechanism, for structural simplicity and energy efficiency. At the same time, during normal flight, it is necessary to ensure that both wings are in synchronization. Combining the above requirements, this paper gives the following three options [25].

(1) Single crank slider mechanism, as shown in Figure 2(a) below. This mechanism can ensure the synchronization of the two wings, and it is convenient to adjust the angle and speed of the flap. However, the slider mechanism will lead to higher friction and energy consumption.

(2) Single crank and double rocker mechanism, as shown in Figure 2(b) below. Compared with the first mechanism above, this flapping mechanism has high efficiency of energy conversion. But it is difficult to ensure movement synchronization of two wings on each side of the air vehicle.

(3) Double crank and double rocker mechanism, as shown in Figure 2(c) below. This mechanism is an improved solution based on the second one. While inheriting the high efficiency of energy transmission of the second mechanism, the use of synchronized double cranks can ensure the synchronization of the two wings during the flapping flight.

The third mechanism integrates the advantages of the first two mechanisms with only a small increase in the energy consumption between the gears. Therefore, the design in this paper selects the third mechanism as the flapping-wing linkage mechanism, which will be further optimized in the following sections.

3.2. Linkage Size Calculation for Flapping Wing. Figure 3 below shows the flapping-wing configuration of the right half (since the two flaps are symmetrical, the results of the analysis here still apply to the left half). The design requires the upward and downward flapping motion of the large wing (CD) as crank (a) rotates at a constant speed. In addition, the change of angle between the large and small wings is also desired to generate variable wingspan during the up-anddown strokes, so that a lift force can be generated [26, 27]. Therefore, it is necessary to determine the length of each 


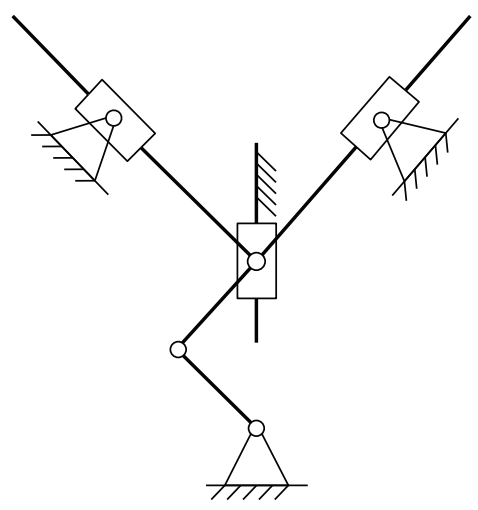

(a)

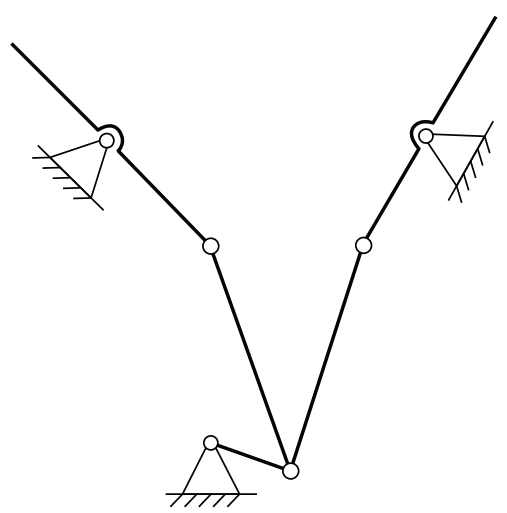

(b)

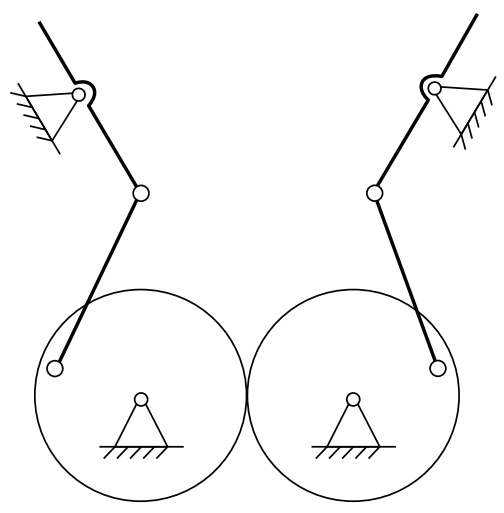

(c)

FIgURE 2: Flapping-wing mechanisms. (a) Single crank slider. (b) Single crank double rocker. (c) Double crank double rocker.

connecting rod to ensure the variation of angle $\beta$ and angle $\gamma$ as shown in Figure 3.

Assume that the coordinate is $(m, n)$ for point $C$ and $(a * \cos \alpha, a * \sin \alpha$, ) for point $A$. Then, in $\triangle \mathrm{ABC}$, we have

$$
\cos \gamma=\frac{b^{2}+c^{2}-d^{2}}{2 b c}
$$

In addition, the followings hold:

$$
\begin{aligned}
& d=(m-a \cos \alpha)^{2}+(n-a \sin \alpha)^{2}, \\
& \gamma=\cos ^{-1} \frac{b^{2}+c^{2}-(m-a \cos \alpha)^{2}-(n-a \sin \alpha)^{2}}{2 b c},
\end{aligned}
$$

$$
\begin{aligned}
\beta+\angle \mathrm{ACB}+\angle \mathrm{AC} y & =\frac{\pi}{2}, \\
\frac{b}{\sin \angle \mathrm{ACB}} & =\frac{d}{\sin \gamma},
\end{aligned}
$$

where angle $\gamma=\angle \mathrm{EBC}$ in the parallelogram EBDF, which represents the relative angle between small wing (DG) and large wing (DB).

Combining equations (1)-(5), the angle of large wing $\beta$ can be obtained by

$$
\beta=\frac{\pi}{2}-\tan ^{-1} \frac{m-a \cos \alpha}{n-a \sin \alpha}-\sin ^{-1}\left(\frac{b}{\sqrt{(m-a \cos \alpha)^{2}+(n-a \sin \alpha)^{2}}} \sin \gamma\right)
$$

In the above equation, the rotation angle of the crank $\alpha$ is the independent variable, and the $\gamma$ and $\beta$ are the function values. Now, it is necessary to determine the values of the five linkage parameters $a, b, c, d$, and $e$. Linkage $\mathrm{A}$ is actually a simplified gear, so the size of the gear should be roughly determined before determining the length of the rod. In this paper, the number of teeth of gear $\mathrm{A}$ is 40 , and the modulus is 0.5 , so the diameter of the gear is $20 \mathrm{~mm}$. Here, the length of crank (a) is $7.5 \mathrm{~mm}$. For crank (a) to rotate continuously, the following inequality must hold:

$$
b+c-a>\sqrt{m^{2}+n^{2}} .
$$

Taking $b=c=15$, the MATLAB calculation shows that when $m=6.5$ and $n=13$, an optimal flutter curve can be obtained, which is illustrated in Figure 4 below. Here, the angle of large wing $\beta$ varies within the range of $-36^{\circ}$ to $+34^{\circ}$, generating a near symmetrical flapping movement up and down during flight. Moreover, the upward stroke of the large wing takes a longer time than the down stroke (as shown by the slope difference), which is helpful for producing lift force. To design the small wing mechanism for efficient flapping, $\angle F D G$ needs to be optimized, so that the angle between the large and small wings (i.e., $\phi=\gamma-\angle \mathrm{FDG}$ ) follows a coordinated motion. By trial and error, it is found that when $\angle \mathrm{FDG}=30^{\circ}$ approximately, the flapping motion of the mechanism is very close to the bird's bionic wing movements as shown in Figure 1. During the down stroke, the angle of the small wing $\theta$ is almost the same as the large wing $\beta$, which represents the collinear flapping movement. During the folding stroke, the angle between the small wing and large wing (i.e., $\phi=\beta-\theta$ in Figure 4(b)) increases, generating the folding effect. During lift stroke, the angle difference $\phi$ stays almost constant (i.e., the small wing and large wing flap almost together), while the angle of large wing $\beta$ keeps increasing. During the unfolding stroke, the angle of large wing $\beta$ stays almost unchanged, while the angle of small wing $\theta$ keeps increasing until it reaches the large wing. The four flapping strokes form a complete flapping cycle of the flight, which corresponds to one full revolution of the crank, as shown in Figure 4.

\subsection{Mechanism Design}

3.3.1. Wing Skeleton Design. The wing cross section of the micro flapping-wing robot should be similar to that of a bird. In order to reduce the mass of the whole air vehicle, this paper designs the skeleton structure of the large and small wings by using wing skeletons arranged at intervals and covering the outside with a lightweight film. The large wing is not designed for pitching motion because of its close proximity to the fuselage, so the large wing is designed to 


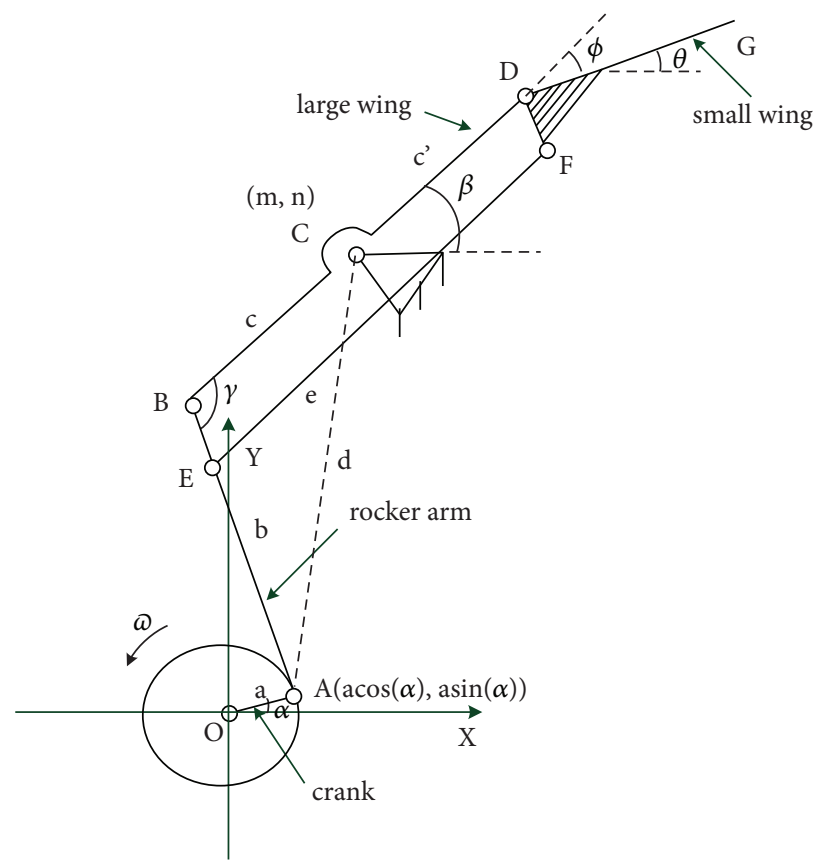

Figure 3: Kinematic analysis of the flapping wing.

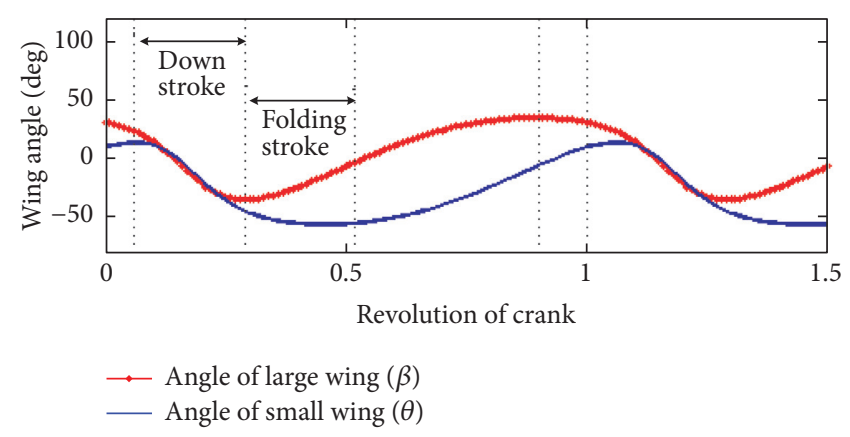

(a)

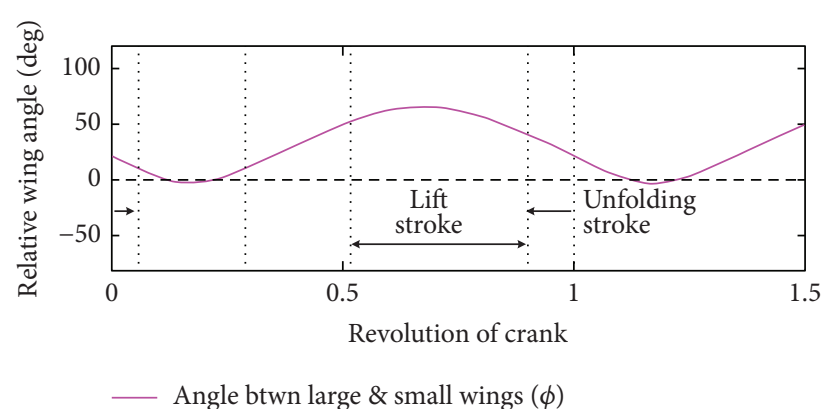

(b)

Figure 4: Time history of wing angles during flapping flight. (a) Wing angles of large and small wings. (b) Relative wing angle between large and small wings.

flutter horizontally at all times. As the structure of the large wing skeleton is shown in Figure 5 below, only a small amount of thrust can be generated during the upward fluttering process, which is almost negligible. The basic structure of the small wing skeleton is similar to that of the large wing, but in order to generate pitching motion, its connection with the connecting rod needs to be designed with a circular hole. The big wing needs to consider the movement of rod $e$ (i.e., the longer side of the parallelogram that drives the small wing to generate the angle in Figure 3) in the vertical axis. Furthermore, the skeleton of the big wing needs to be designed wider so that there is enough space inside for the rod $e$ to move up and down. In order to strengthen the whole skeleton, some net-like connection parts are created inside the skeleton to make it less deformable and stiffer. The design of the wing skeletons is shown in Figures 5 and 6 below.
3.3.2. Pitch Mechanism Design. To achieve the ability to fly, a micro flapping-wing robot requires not only lift but also thrust. The main source of thrust for a bird in flight is the ability of the wings to pitch with the airflow. Therefore, we need the small wing to be able to rotate around the leading edge of the wing during the fluttering process.

In this paper, the pitching motion is designed from the mechanism itself. In the upward stroke, if the wing can tilt slightly around the leading edge toward the ground, the air force on the wing will have a forward component, which generates the thrust. Moreover, the upward stroke is also accompanied by the decreasing of the relative angle between the big wing and small wing, and in the downward stroke, the angle between the big wing and small wing will return to the maximum value. The pitching mechanism used in this design is shown in Figure 6 below. When the angle of the small wing and the large wing is close to $180^{\circ}$, point $G$ is 


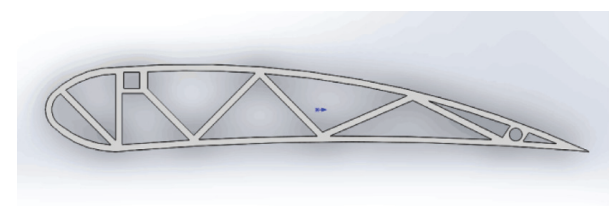

FIGURE 5: Large wing skeleton.

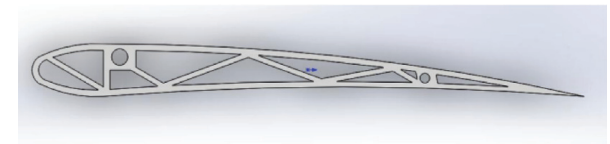

FIgURE 6: Small wing skeleton.

closest to point $H$, and the bar parallel to the plane of the skeleton is kept straight, or slightly upward. When the angle of the big wing and small wing becomes smaller (i.e., during the upward stroke), the distance between point $G$ and point $H$ will become larger, resulting in a tension between point $I$ and point $J$. Here, point $I$ is almost kept at the same level as point $G$. As the ball hinge pulls the $\operatorname{rod} g$ downward, $\operatorname{rod} g$ is tilted around the rod $f$ in the direction of the ground to achieve the desired pitching. A detail of the pitching mechanism is shown in Figure 7 , and the corresponding pitching effect during up-and-down strokes is shown in Figure 8 below.

3.3.3. Fuselage and Tail Mechanism Design. In order to ensure that the weight of the whole aircraft is as low as possible, the fuselage designed in this paper is hollow in the middle and skinned in the outer layer. To ensure the connection between the wing skeleton and the pitch mechanism on the fuselage, the fuselage is connected with a thick carbon fibre rod in the central axle to ensure the relative position of each fuselage skeleton. The fuselage design is shown in Figure 9, with a circular basic shape and pentagonal support bars.

The tail plays an important role in stabilizing and steering the flapping-wing air vehicle. The pitch, yaw, and roll motions of birds are achieved by differential wing flapping, coupled with the control of the tail. However, the flapping mechanism designed above has difficulty in achieving differential flapping of the wings (as the flapping motion on both sides is in strict synchronization), so controlling the direction and changing the flight attitude are only achieved by the tail. Inspired by the tail shape of a bird, the tail of the flapping-wing robot is designed to be fanshaped, truncated at the bottom, and has three mounting holes to fit the fuselage, as shown in Figure 10 below. The structure is skeletonized and skinned on the outside to reduce mass and improve flight efficiency.

During the flight, aerodynamic forces are applied on the tail, which generates a moment around the center of gravity due to the tail force arm. The moment generated by the tail can make the whole flapping-wing robot reach the moment balance. With proper control of the tail, the aerodynamic effect of the whole flapping-wing air vehicle can always be shifted to the center of gravity, which contributes to flight stability. The deflection of the tailplane will generate a maneuvering moment that will allow the flapping-wing robot to change its attitude or to maintain balance in different flight attitudes, as illustrated in Figure 11.

\section{Overall Design and Motion Analysis}

4.1. Overall Design. The bionic design and 3D rendering of the flapping-wing air vehicle are shown in Figure 12 below. Figure 12(a) shows the FWAV with the structural elements, including batteries, gears, and servos. Figure 12(b) illustrates the FWAV with thin-film skin wrapped around its fuselage, wings, and tail, for aerodynamic performance. Geometrically, the distance between the furthest points of the wings is $318 \mathrm{~mm}$ with the wings extended, and the fuselage length is $192 \mathrm{~mm}$. Most of the structural parts are made of carbon fibre, and some noncritical materials are made of ABS plastic to further reduce the weight of the whole air vehicle. The connecting rods are connected to each other by bearings, and the two rods are connected by the interference fit of the bearing caps. To reduce the stress on the connecting rod, a support rod is added at the rear of the skeleton.

4.2. Analysis of Flapping Motion. Based on the kinematic analysis of the flapping mechanism, the curves for flapping motion can be obtained. Figure 13 below shows the displacement curve of the small wing tip in the vertical direction, and Figure 14 shows that in the horizontal wingspan direction. From these two figures, it can be seen that the flutter period is $2 \mathrm{~s}$. This period is set for the convenience of simulation and animation, which is not the flapping period of the micro flapping-wing robot in realworld operation. Since the actual speed of the flapping motion is much faster than that in simulation, the simulation results in Figures 13 to 18 are the slowed-down versions. As we can see from the figure below, the tip of the small wing moves up to the highest point and down to the lowest point in one cycle. The upward stroke takes a longer time $\left(T_{U}=1.2 \mathrm{~s}\right)$, and the downward stroke takes a shorter time $\left(T_{D}=0.8 \mathrm{~s}\right)$. The time ratio of upward and downward is $3: 2$, which produces a positive lift force in each flapping cycle.

The displacement in the horizontal wingspan direction at the end of the flap is largely equal to the change in the wingspan area during the flap $[28,29]$. As can be seen in Figure 14, the tip of the small wing undergoes a folding to unfolding during both the up-and-down strokes. In each stroke, when the wing is in the horizontal position, the wingspan area attains the largest value in the whole stroke.

By plotting the vertical and horizontal displacements in the $X Y$ plane, the small wing tip trajectory is obtained, as shown in Figure 15. The wing tip trajectory contains both the down stroke and up stroke portion, where the associated flapping direction is indicated by the arrow. Comparing the wingspan (in $X$-direction) of the up-and-down strokes, it can be seen that the wingspan during down stroke is always larger than up stroke. Such behaviour also contributes to 


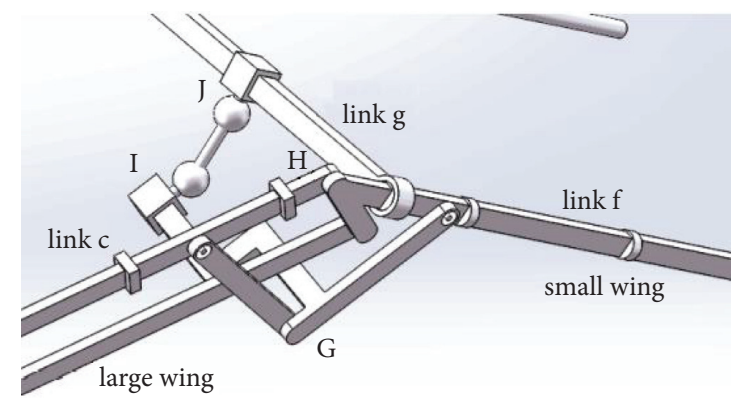

FIGURE 7: Pitching mechanism between the large wing and small wing.

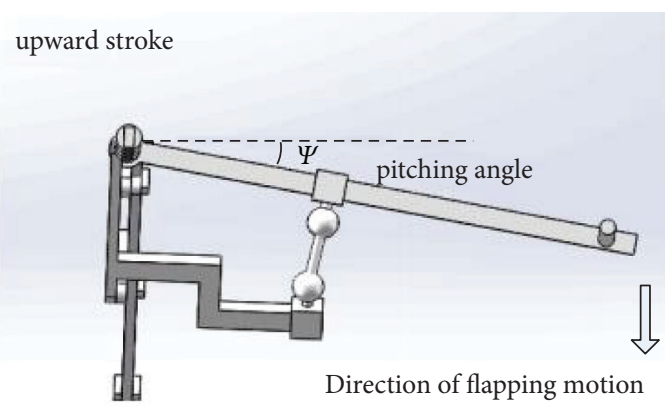
downward stroke

Figure 8: Pitching angle of the small wing during up-and-down strokes (side view).

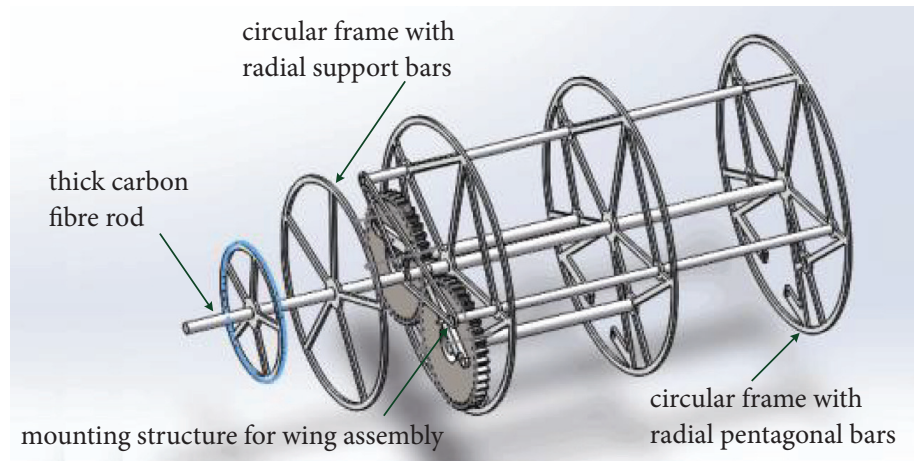

FIGURE 9: Fuselage structure.

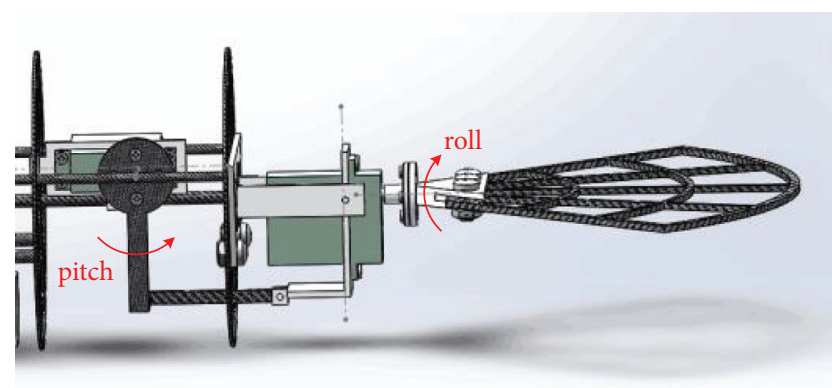

FIGURE 10: Tail structure with both pitch and roll freedom.

positive aerodynamic lift during the flapping flight of the air vehicle [30].

The formula for air resistance shows that the resistance of an object in motion is proportional to the windward area of the object and proportional to the square of the velocity
$[31,32]$. The windward area of the wing is related to the displacement of the horizontal wingspan at the end of the flap in Figure 14, and the velocity is related to the velocity of the flap end point. The velocity curves of the small wing tip are shown in Figures 16 and 17 below.

4.3. Pitch Motion Analysis. Through the kinematic simulation, it can be seen that the flapping-wing air robot will make a pitching motion around the leading edge during the lifting stroke. To illustrate the pitching motion quantitatively, it can be represented by the angle change between the pitching motion lever and the upper plane of the armlet linkage. The time history of the pitch angle for the small wing is shown in Figure 18 below.

From Figure 18, it is seen that the small wing performs the pitching motion with an amplitude of about 10 degrees $(p k-p k)$, in addition to flapping movement during the flight. Combining Figures 18 with 13, 19 is obtained to 


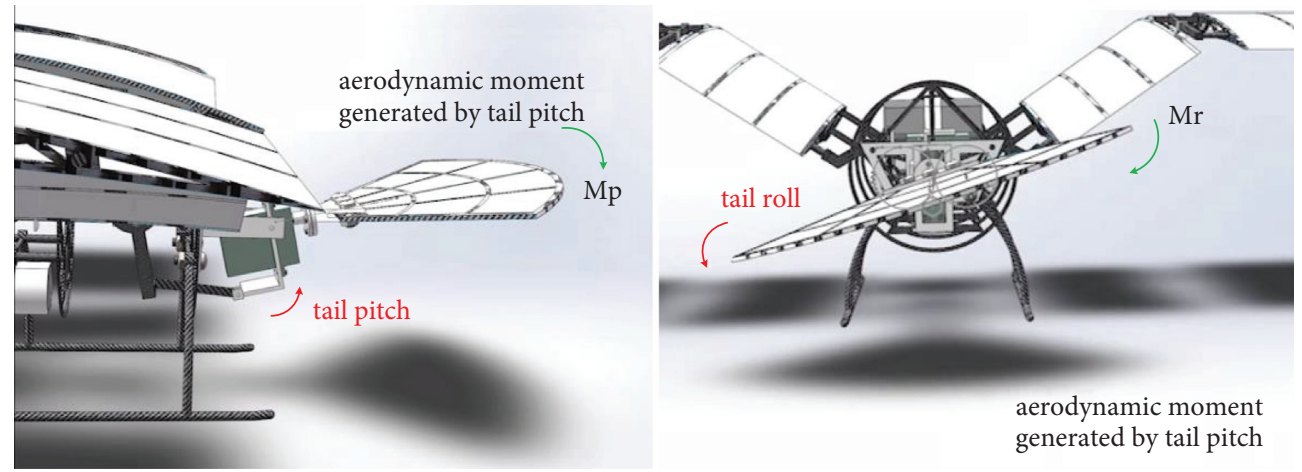

FIGURE 11: Aerodynamic moment generated by tail control.

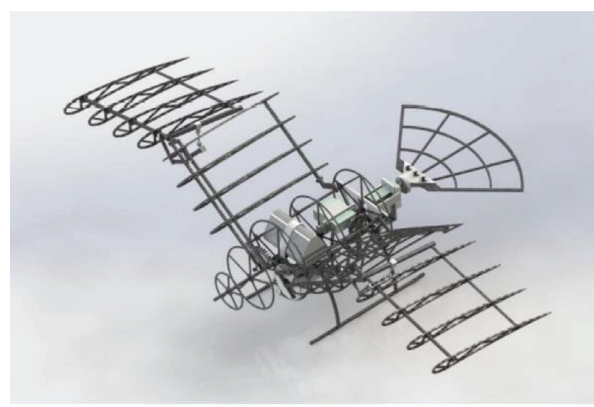

(a)

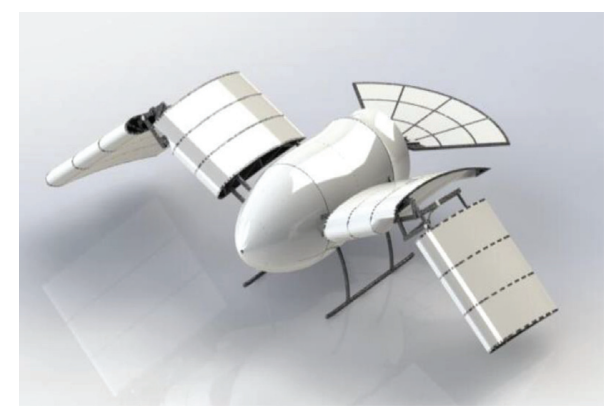

(b)

FIGURE 12: 3D rendering of the overall structure of the FWAV. (a) FWAV with skeleton shown. (b) FWAV with skin covered.

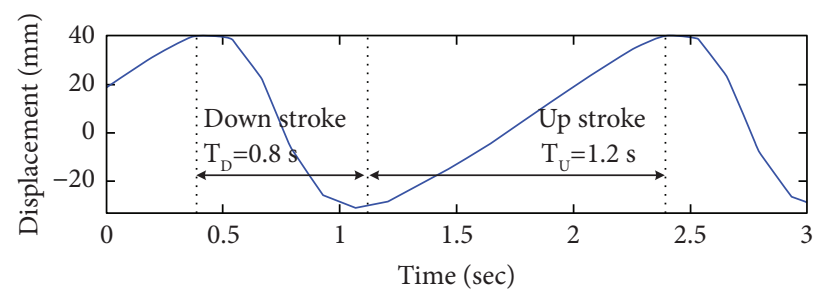

Figure 13: Vertical displacement at the small wing tip.

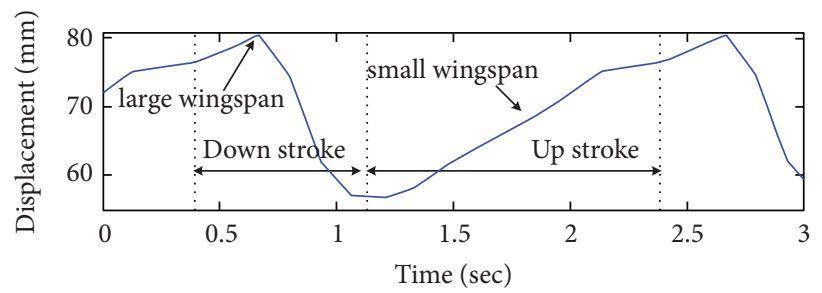

Figure 14: Horizontal displacement at the small wing tip.

demonstrate the coordinated pitching movement with the flapping motion, where the blue curve is the displacement of the small wing tip in the vertical direction during the flap, and the red curve is its pitch angle. It can be seen that during the lift stroke, the tip of the small wing moves upward (blue curve), and the pitch angle of the small wing increases (red curve) for lift generation. During the downstroke, the pitch angle stays around $7^{\circ}$, with only very small fluctuations of 


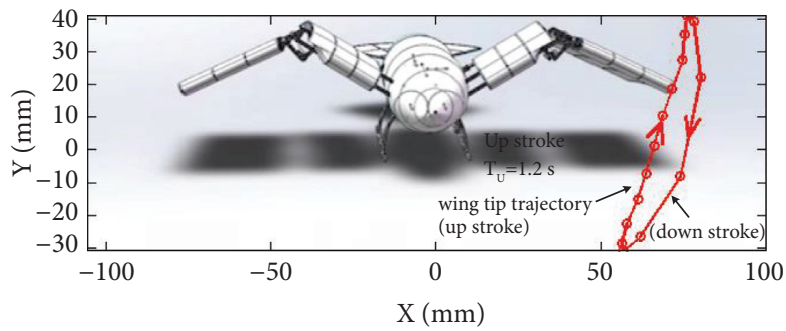

FIGURE 15: Small wing tip trajectory during the whole flap (front view).

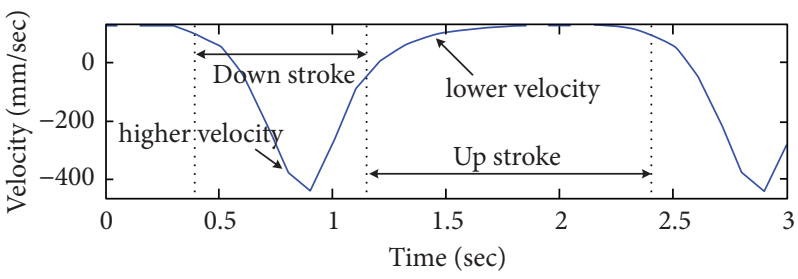

Figure 16: Vertical velocity at the small wing tip.

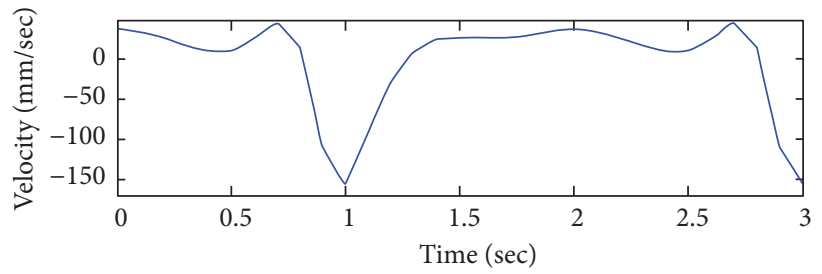

FIGURE 17: Horizontal velocity of small wing tip.

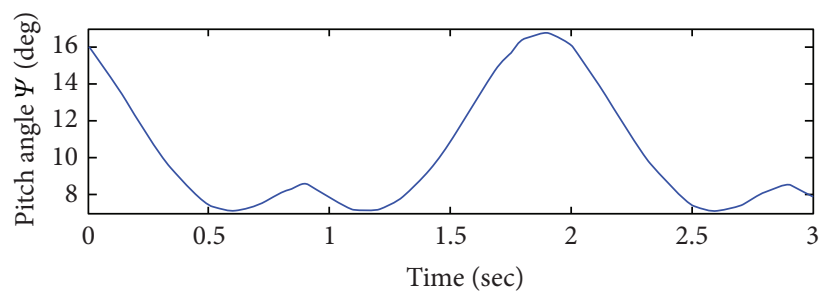

FIgURe 18: Pitch angle of the small wing.

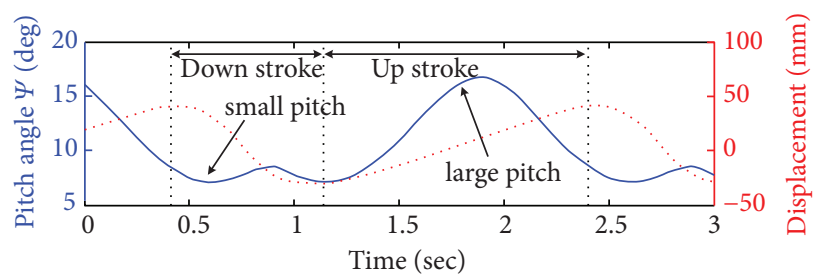

Figure 19: Pitch angle and vertical displacement of the small wing. 
$\pm 0.5^{\circ}$. Namely, the pitch motion rod and the upper plane of the boom linkage are almost at the same level, to minimize the pitch and maximize effective wingspan area for down stroke [33].

\section{Conclusion}

Aiming at structural simplicity, motion symmetry, and flapping efficiency, this paper proposes an optimized double crank and double rocker mechanism as the flapping mechanism. Proper flapping-wing size is determined by analyzing the relative position between the driving mechanism and the flapping mechanism. Moreover, a novel pitch mechanism consisting of ball hinges is designed to realize the small wing's pitching motion for additional thrust. The fuselage and landing gear of the flapping-wing robot are designed for low air resistance and lightweight, and the tail mechanisms are designed to have both roll and pitch freedoms, offering advanced manoeuvrability during flight. Simulation results show faster flapping motion with larger wingspan area during the downstroke than upstroke, for positive aerodynamic lift. Additionally, the novel pitching design for the small wing further improves the lift forces during flight. The research in this paper is an important resource for novice researchers to design and study the advanced and complex problems of flapping-wing air vehicles.

\section{Data Availability}

The data used to support the findings of this study are available from the corresponding author upon request.

\section{Conflicts of Interest}

The authors declare that they have no conflicts of interest.

\section{Acknowledgments}

This research was funded by the National Natural Science Foundation of China (NSFC grant nos. 61903081 and 51875101), Natural Science Foundation of Jiangsu Province (grant no. BK 20170690), Jiangsu Province Key R\&D Program (grant no. BE 2021730), and Zhishan Youth Scholar Program of Southeast University (grant no. 2242021R41135).

\section{References}

[1] M. Liu, D. Ma, and S. Li, "Neural dynamics for adaptive attitude tracking control of a flapping wing micro aerial vehicle," Neurocomputing, vol. 456, pp. 364-372, 2021.

[2] H. Isakhani, C. Xiong, W. Chen, and S. Yue, "Towards locustinspired gliding wing prototypes for micro aerial vehicle applications," Royal Society Open Science, vol. 8, no. 6, Article ID 202253, 2021.

[3] X. Wei, D. Li, J. Jiaqi, T. Puxue, and X. Jinwu, "Experimental and numerical study of flapping wing rotary MAV," in Proceedings of the 2017 IEEE International Conference on Unmanned Systems (ICUS), Unmanned Systems (ICUS), pp. 23-28, Beijing, China, October 2017.
[4] W. He, T. Meng, X. He, and C. Sun, "Iterative learning control for a flapping wing micro aerial vehicle under distributed disturbances," IEEE Transactions on Cybernetics, vol. 49, no. 4, pp. 1524-1535, 2019.

[5] T. Wang, S. Jin, and Z. Hou, "Model free adaptive pitch control of a flapping wing micro aerial vehicle with input saturation," in Proceedings of the 2020 IEEE 9th Data Driven Control and Learning Systems Conference (DDCLS), pp. 627-632, Liuzhou, China, November 2020.

[6] T. N. Pornsin-Sirirak, Y. C. Tai, C. M. Ho, and M. Keennon, "Microbat a palm-sized electrically powered ornithopter," in Proceedings of the nasa/jpl workshop on biomorphic robotics, Pasadena, CA, USA, August 2001.

[7] M. Okamoto, K. Yasuda, and A. Azuma, "Aerodynamic characteristics of the wings and body of a dragonfly," Journal of Experimental Biology, vol. 199, no. 2, pp. 281-294, 1996.

[8] M. Sato and A. Azuma, "The flight performance of a damselfly Ceriagrion melanurum Selys," Journal of Experimental Biology, vol. 200, no. 12, pp. 1765-1779, 1997.

[9] M. F. M. Osborne, "Aerodynamics of flapping flight with application to insects," Journal of Experimental Biology, vol. 28, no. 2, pp. 221-245, 1951.

[10] J. T. C. Yeh and K. R. Grebe, "Patterning of poly-paraxylylenes by reactive ion etching," Journal of Vacuum Science and Technology A: Vacuum, Surfaces, and Films, vol. 1, no. 2, pp. 604-608, 1983.

[11] D. Klavdianos, "Aeria Bird Repelling System. From lab to the landing strip. From the FESTO SmartBird towards a viable product against bird strikes," Master Thesis, Delft University of Technology, The Netherlands, 2014.

[12] W. Send, "SmartBird-Vogelmodell mit effizientem Biegetorsionsantrieb," Luft- und Raumfahrt, vol. 5, pp. 26-28, 2011.

[13] W. Z. Cai, "The current trends of researches on entomopters," ENTOMOLOGICAL KNOWLEDGE, vol. 39, no. 6, pp. 464455, 2002.

[14] W. Sochacki and D. Cekus, "The new concept of power transmission to the entomopter wings," Journal of Micro-Bio Robotics, vol. 16, no. 2, pp. 225-235, 2020.

[15] F. Mazhar and S. I. A. Shah, "On the unsteady aerodynamics and design of flapping wing vehicles," in Proceedings of the 17th International Bhurban Conference on Applied Sciences and Technology (IBCAST), pp. 110-121, Islamabad, Pakistan, January 2020.

[16] M. L. Hu, R. X. Wei, and X. F. Cui, "Inertia force of flapping wing in entomopter micro air vehicle," Journal of Aerospace Power, vol. 23, no. 7, pp. 1279-1286, 2008.

[17] M. Wu, Z. Shi, T. Xiao, and H. Ang, "Energy optimization and investigation for Z-shaped sun-tracking morphing-wing solarpowered uav," Aerospace Science and Technology, vol. 91, pp. 1-11, 2019.

[18] M. Wu, Z. Shi, T. Xiao, Z. L. J. Chen, and H. Ang, "Effect of solar cell efficiency and flight condition on optimal flight control and energy performance for Z-shaped wing stratospheric solar aircraft," Acta Astronautica, vol. 164, pp. 366375, 2019.

[19] M. Wu, T. Xiao, H. Ang, and H. Li, “Optimal flight planning for a Z-shaped morphing-wing solar-powered unmanned aerial vehicle," Journal of Guidance, Control, and Dynamics, vol. 41, no. 2, pp. 497-505, 2018.

[20] D. Wei, Z. Shi, X. Geng, and H. Ang, "Experimental investigation of wing-body rock with nonzero equilibrium roll angles," Proceedings of the Institution of Mechanical Engineers - Part G: Journal of Aerospace Engineering, vol. 232, no. 4, pp. 771-782, 2018. 
[21] T. H. Xiao, D. M. Luo, X. M. Zheng, H. S. Ang, and A. H. Ji, "Progress and challenges in unsteady aerodynamic optimization design of bionic flapping-wings," Acta Aerodynamica Sinica, vol. 36, no. 1, pp. 80-87, 2018.

[22] H. Li, L. Liu, T. Xiao, and H. Ang, "Design and simulative experiment of an innovative trailing edge morphing mechanism driven by artificial muscles embedded in skin," Smart Materials and Structures, vol. 25, no. 9, 2016.

[23] Q.-V. Nguyen, W. L. Chan, and M. Debiasi, "Hybrid design and performance tests of a hovering insect-inspired flappingwing micro aerial vehicle," Journal of Bionics Engineering, vol. 13, no. 2, pp. 235-248, 2016.

[24] L. Liu, Z. Fang, and Z. He, "Optimization design of flapping mechanism and wings for flapping-wing MAVs," in Proceedings of the 1st International Conference on Intelligent Robotics and Applications (ICIRA), pp. 245-255, Wuhan, China, October 2008.

[25] Z. Yafeng, Y. Chunming, Z. Xvhui, Y. Liang, and Q. Jvxin, "The optimum design of the flapping-wing mechanism," IOP Conference Series: Materials Science and Engineering, vol. 631, no. 3, 2019.

[26] R. Madangopal, Z. A. Khan, and S. K. Agrawal, "Biologically inspired design of small flapping wing air vehicles using fourbar mechanisms and quasi-steady aerodynamics," Journal of Mechanical Design, vol. 127, no. 4, pp. 809-816, 2004.

[27] Y. Zhang, X. Wang, G. Zhang, and J. Yang, "Design and analysis of single-degree-of-freedom flapping wing mechanism based on UG," Journal of Physics: Conference Series, vol. 1629, no. 1, p. 7, Article ID 012060, 2020.

[28] M. L. Anderson and R. G. Cobb, "Toward flapping wing control of micro air vehicles," Journal of Guidance, Control, and Dynamics, vol. 35, no. 1, pp. 296-308, 2012.

[29] V. M. Mwongera, "A Review of flapping wing MAV modelling," International Journal of Aeronautical Science \& Aerospace Research, vol. 2, no. 2, pp. 27-36, 2015.

[30] T. M. Yang and F. Y. Hsiao, "Dynamics of flapping micro aerial vehicles," in Proceedings of the American Control Conference, pp. 4190-4195, IEEE Publications, St. Louis, MO, USA, June 2009.

[31] G. K. Taylor and A. L. R. Thomas, "Animal flight dynamics II: longitudinal stability in flapping flight," Journal of Theoretical Biology, vol. 214, no. 3, pp. 315-370, 2002.

[32] X. Xinyan Deng, L. Schenato, W. C. Wei Chung Wu, and S. S. Sastry, "Flapping flight for biomimetic robotic insects: part I-system modeling," IEEE Transactions on Robotics, vol. 22, no. 4, pp. 776-788, 2006.

[33] S. P. Sane and M. H. Dickinson, "The control of flight force by a flapping wing: lift and drag production," Journal of Experimental Biology, vol. 204, no. 15, pp. 2607-2626, 2001. 